\title{
Oxaprozin-Induced Apoptosis on CD40 Ligand-Treated Human Primary Monocytes Is Associated with the Modulation of Defined Intracellular Pathways
}

\author{
Fabrizio Montecucco, ${ }^{1}$ Maria Bertolotto, ${ }^{2}$ Luciano Ottonello, ${ }^{2}$ \\ Alessandra Quercioli, ${ }^{2}$ François Mach, ${ }^{1}$ and Franco Dallegrii ${ }^{2}$ \\ ${ }^{1}$ Division of Cardiology, Faculty of Medicine, Foundation for Medical Researches, University Hospital of Geneva, \\ 1211 Geneva, Switzerland \\ ${ }^{2}$ Clinic of Internal Medicine I, Department of Internal Medicine, Medical School, University of Genoa, \\ 16143 Genoa, Italy
}

Correspondence should be addressed to Fabrizio Montecucco, fabrizio.montecucco@medecine.unige.ch

Received 16 February 2009; Revised 2 June 2009; Accepted 17 June 2009

Recommended by Mostafa Z. Badr

The modulation of CD40L activity might represent a promising therapeutic target to reduce monocyte inflammatory functions in chronic diseases, such as rheumatoid arthritis. In the present study, we investigated the possible influence of nonsteroidal anti-inflammatory drugs (NSAIDs) on CD40L-induced monocyte survival. Monocytes were isolated from buffy coats by using Ficoll-Percoll gradients. Monocyte apoptosis was evaluated by fluorescence microscopy on cytopreps stained with acridine orange or using flow cytometry analysis of Annexin- $\mathrm{V}$ and Propidium Iodide staining. Akt and NF- $\kappa \mathrm{B}$ activation was assessed using western blot. Caspase 3 activity was determined spectrophotometrically. Among different NSAIDs, only oxaprozin dosedependently increased apoptosis of CD40L-treated monocytes. Oxaprozin pro-apoptotic activity was associated with the inhibition of CD40L-triggered Akt and NF- $\kappa \mathrm{B}$ phosphorylation and the activation of caspase 3 . In conclusion, our data suggest that oxaprozin-induced apoptosis in CD40L-treated human monocytes is associated with previously unknown cyclooxygenase (COX)independent pathways. These intracellular proteins might be promising pharmacological targets to increase apoptosis in CD40Ltreated monocytes.

Copyright (C) 2009 Fabrizio Montecucco et al. This is an open access article distributed under the Creative Commons Attribution License, which permits unrestricted use, distribution, and reproduction in any medium, provided the original work is properly cited.

\section{Introduction}

Human monocytes are central players in the pathogenesis of chronic inflammatory disorders, such as atherosclerosis and rheumatoid arthritis [1-3]. Monocytes are immune cells circulating in the blood stream, which are recruited to inflammatory sites for the immune response $[2,4]$. Once resident in inflamed tissues, they can differentiate into several cell types, such as macrophages, dendritic cells, and also osteoclast-like cells [5-7] contributing to further increase inflammation. In this context, the induction of monocyte apoptosis with the consequent reduction of inflammatory cell infiltrates has to be considered as a crucial target to reduce inflammatory disease persistence. CD40 ligand
(CD40L) is a crucial mediator in monocyte functions and differentiation [8]. Thus, the modulation of CD40L-induced proinflammatory activities could represent a very promising therapeutic strategy to reduce monocyte-mediated tissue infiltration and injury. In the present article, we planned to investigate the possible influence of nonsteroidal antiinflammatory drugs (NSAIDs) on CD40L-treated monocyte apoptosis $[9,10]$. In particular, we focused on oxaprozin, an achiral oxazole-propionic acid derivative (4,5-diphenyl2-oxazolepropionic acid) that has been recently shown to modulate monocyte survival in inflammatory microenvironments [11]. In order to identify the possible molecular mechanisms involved in, we studied the role of different intracellular mediators, such as cyclooxygenase 
(COX), phosphoinositol-3 kinase (PI3K)/Akt, extracellular signal-regulated kinase (ERK) 1/2, p38 mitogen activated kinase (MAPK), nuclear factor kappaB (NF- $\kappa \mathrm{B})$, and caspase 3.

\section{Materials and Methods}

2.1. Human Monocyte Isolation. Human monocytes were isolated from buffy coats, obtained from healthy volunteers, under a protocol approved by the local Ethics Committee. All donors provided written, informed consent to the procedure and use of the cells. After centrifugation on a Ficoll-Hypaque density gradient, mononuclear cells were collected from the interface and washed with PBS. Monocytes were then purified from the upper interface of a hypotonic Percoll density gradient $(1.129 \mathrm{~g} / \mathrm{mL})$. Purified monocytes were resuspended in RPMI 1640 medium supplemented with 1\% $(\mathrm{v} / \mathrm{v})$ heat-inactivated fetal calf serum (FCS) and $500 \mathrm{ng} / \mathrm{mL}$ polymyxin B, as previously described [12]. At the end of purification, viability of monocytes was more than $98 \%$, as determined by ethidium bromide-fluoresceine diacetate assay (from Sigma-Aldrich S.r.l., Milano, Italy). Monocyte purity was at least $90 \%$, as assessed by flow cytometric analysis (staining with FITC-conjugated antihuman CD14 antibody $(\mathrm{Ab})$, from $\mathrm{BD}$ Pharmingen, Franklin Lakes, NJ) and nonspecific esterase staining.

2.2. Assessment of Monocyte Apoptosis. Purified monocytes were resuspended at $10^{6} / \mathrm{mL}$ and cultured for 48 hours in culture medium containing RPMI 1640 with 25 mM HEPES (Irvine Scientific, Santa Ana, CA), $500 \mathrm{ng} / \mathrm{mL}$ polymixin B (Sigma-Aldrich), and 1\% heat-inactivated FCS (ICN Biomedicals s.r.l., Milano, Italy) in the presence or absence of $200 \mathrm{ng} / \mathrm{mL}$ CD $40 \mathrm{~L}$ plus $1 \mu \mathrm{g} / \mathrm{mL}$ CD $40 \mathrm{~L}$ enhancer (both from Alexis Biochemicals, Carlsbad, CA). In selective experiments, cells were cultured in the presence or absence of $50 \mu \mathrm{M}$ PD98059 (MEK inhibitor, a kinase activating ERK 1/2, from Biomol Research Laboratories, Inc., Plymouth Meeting, PA), $1 \mu \mathrm{M}$ SB203580 (p38 MAPK inhibitor, from Biomol Research Laboratories, Inc.), $50 \mu \mathrm{M}$ LY294002 (PI3K inhibitor, from Sigma-Aldrich), $20 \mu \mathrm{M}$ SN-50 (NF$\kappa \mathrm{B}$ inhibitor, from Calbiochem, San Diego, CA), $50 \mu \mathrm{M}$ Ac-DEVD-CHO (caspase 3 inhibitor, from Bachem AG, Bubendorf, Switzerland), different doses (5, 10, 50, $100 \mu \mathrm{M})$ of oxaprozin (Helsinn Healthcare SA, Lugano, Switzerland), $100 \mu \mathrm{M}$ ibuprofen (Sigma-Aldrich), $100 \mu \mathrm{M}$ indomethacin (Sigma-Aldrich), or $100 \mu \mathrm{M}$ naproxene (Sigma-Aldrich). Percentages of apoptotic cells were measured by both fluorescence microscope (staining with acridine orange from Sigma-Aldrich and Annexin V-FITC Kit, from MBL International, Woburn, MA) and flow cytometer (by using Propidium Iodide Kit, from MBL International). Immunofluoresence analysis of Annexin V and Propidium iodide binding was performed by following the manufacturer's instructions with minor changes, as previously described [13].

2.3. Immunoblot Analysis. $10^{7}$ cells $/ \mathrm{mL}$ monocytes were prencubated in the presence or absence of $100 \mu \mathrm{M}$ oxaprozin for 1 hour in a humidified atmosphere $5 \% \mathrm{CO}_{2}$ at $37^{\circ} \mathrm{C}$ and then, without washing, stimulated in the presence or absence of $200 \mathrm{ng} / \mathrm{mL}$ CD $40 \mathrm{~L}$ plus $1 \mu \mathrm{g} / \mathrm{mL}$ CD $40 \mathrm{~L}$ enhancer, for 15 minutes. The reaction was stopped on ice, and cells were centrifuged at $4^{\circ} \mathrm{C}$ to remove culture supernatants. Total protein was extracted in lysis buffer containing $20 \mathrm{mM}$ Tris $\cdot \mathrm{HCl}$ (pH 7.5), $150 \mathrm{mM} \mathrm{NaCl}, 10 \mathrm{mM} \mathrm{NaF}, 1 \%$ Nonidet P-40, $10 \%$ glycerol, $1 \mathrm{mM}$ PMSF, $10 \mu \mathrm{g} / \mathrm{mL}$ aprotinin, $10 \mu \mathrm{g} / \mathrm{mL}$ leupeptin, and $0.5 \mathrm{mM} \mathrm{Na}_{3} \mathrm{VO}_{4}$. Proteins $(40 \mu \mathrm{g})$ were electrophoresed through polyacrylamide-SDS gels and transferred by electroblotting onto nitrocellulose membranes, as previously [14]. Membranes were blocked for 1 hour in 5\% (wt/vol) nonfat milk and then stained with monoclonal antihuman phosphorylated (p)-p65 NFkB, monoclonal antihuman pAkt (both from Santa Cruz Biotechnology, Santa Cruz, CA), polyclonal antihuman p-p38 MAPK, or monoclonal antihuman p-ERK 1/2 Abs (both from R\&D System, Minneapolis, $\mathrm{MN})$.

2.4. Caspase 3 Activity. Caspase 3 activity in the presence or absence of $200 \mathrm{ng} / \mathrm{mL}$ CD $40 \mathrm{~L}$ plus $1 \mu \mathrm{g} / \mathrm{mL}$ CD $40 \mathrm{~L}$ enhancer and $100 \mu \mathrm{M}$ oxaprozin was performed as previously described [15]. The enzymatic activity was spectrophotometrically determined (Titertek TwinReader Plus, Flow Lab, Ltd, Irvine, Scotland) for 60 minutes at $405 \mathrm{~nm}$ assuming an extinction coefficient of $8.8 \times 10^{3} \mathrm{M}^{1} \mathrm{~cm}^{-1}$.

2.5. Statistical Analysis. Statistical analysis was performed by using one-way ANOVA with Bonferroni's posttest (GraphPad InStat version 3.05 for Windows XP, GraphPad Software, San Diego, CA). All data were expressed as mean \pm SEM. Differences were accepted as significant when $P<.05$.

\section{Results}

3.1. Oxaprozin Increases Apoptosis of CD40L-Treated Monocytes. NSAIDs have been previously shown to modulate monocyte functions and monocytic cell survival $[16,17]$. On the other hand, CD40 triggering has been already shown to enhance monocyte survival [9]. Confirming these data, Figure 1(a) shows that $200 \mathrm{ng} / \mathrm{mL}$ CD40L plus $1 \mu \mathrm{g} / \mathrm{mL}$ CD40L enhancer induced a significant $(P<.05)$ reduction of monocyte apoptosis in comparison with control medium. Oxaprozin dose dependently increased CD40Ltreated monocyte apoptosis. $100 \mu \mathrm{M}$ oxaprozin induced the strongest proapoptotic effect. Oxaprozin-mediated activity was also confirmed using Annexin V and Propidium Iodide assays, assessed by fluorescence microscope and flow cytometry (Figure 1(b)). No significant effect on cell apoptosis/death was observed in untreated cells in the presence of different concentrations of oxaprozin (data not shown).

3.2. Oxaprozin Proapoptotic Effect Is Independent of COX Inhibition. To assess the possible role of COX in oxaprozinmediated proapoptotic activity, we studied other NSAIDs, such as ibuprofen, indomethacin or naproxen. To inhibit COX, we used these drugs at higher concentrations $(100 \mu \mathrm{M})$ than their previously reported $\mathrm{IC}_{50}$ for COX-1 and -2 in 


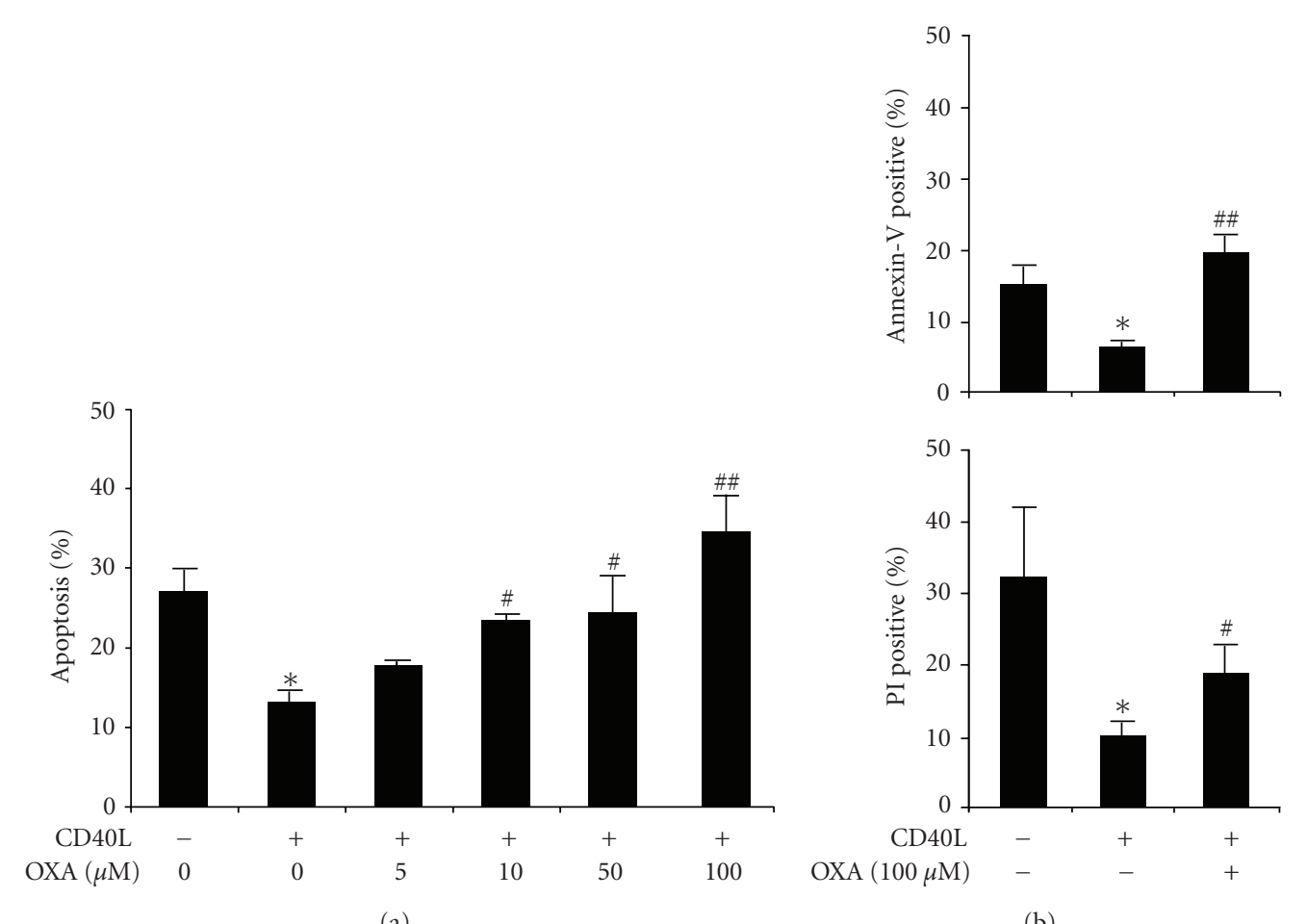

(a)

(b)

FIGURE 1: Oxaprozin increases apoptosis in CD40L-treated monocytes. Monocytes were incubated for 48 hours with control medium or $200 \mathrm{ng} / \mathrm{mL}$ CD 40L plus $1 \mu \mathrm{g} / \mathrm{mL}$ CD40L enhancer in the presence or absence of different doses $(0,5,10,50,100 \mu \mathrm{M})$ of oxaprozin. (a) Assessment of apoptosis by acridine-orange-stained slides under fluorescence microscopy. Data are express as percentages of apoptotic cells on total number of cells counted (mean \pm SEM, $n=3$; apoptosis in absence versus in presence of CD40L: ${ }^{*} P<.05$; apoptosis in presence of CD40L versus CD40L plus 10 or $50 \mu \mathrm{M}$ oxaprozin: ${ }^{\#} P<.05$; apoptosis in presence of CD40L versus CD40L plus $100 \mu \mathrm{M}$ oxaprozin: $\left.{ }^{\#} P<.01\right)$. (b) Assessment of apoptosis by using Annexin V and Propidium Iodide (PI) binding assay. (mean \pm SEM, $n=4$; apoptosis in absence versus in presence of CD40L: ${ }^{*} P<.05$; apoptosis in presence of CD40L versus CD40L plus $100 \mu \mathrm{M}$ oxaprozin: ${ }^{\#} P<.05$ and ${ }^{\# \#} P<.01$ ).

intact cells $[18,19] .100 \mu \mathrm{M}$ oxaprozin significantly increased CD40L-treated monocyte apoptosis. Conversely, treatments with ibuprofen, indomethacin, or naproxene did not induce any effect (Figure 2(a)). These data were also confirmed using Annexin V and Propidium Iodide assay (Figure 2(b)). No significant effect on cell apoptosis/death was observed in untreated cells in the presence of different NSAIDs at $100 \mu \mathrm{M}$ (data not shown).

3.3. Oxaprozin Proapoptotic Activity Is Associated with the Inhibition of CD40L-Triggered Akt and NF- $\kappa B$ Phosphorylation. In order to identify the intracellular pathway involved in oxaprozin-mediated proapoptotic activity, we investigated its possible modulatory effect on CD40L-triggered survival pathways. Figure 3(a) shows that CD40L-mediated reduction of monocyte apoptosis was partially reversed by pretreatment with LY294002 (PI3K inhibitor), PD98059 (MEK 1/2 inhibitor, a kinase activating ERK 1/2), SB203580 (p38 MAPK inhibitor), or SN-50 (NF- $\kappa \mathrm{B}$ inhibitor). In particular, the increase of monocyte apoptosis observed in presence of only one of each selective inhibitor suggesting that all signaling pathway activation is required for CD40Lmediated antiapoptotic effects. Annexin V and Propidium Iodide assays confirmed apoptosis levels morphologi- cally assessed (Figure 3(b)). Oxaprozin treatment inhibited CD40L-induced Akt and NF- $\kappa$ B (p65) phosphorylation (Figure 3(c)). Conversely, a slight increase of ERK 1/2 and p38 MAPK phosphorylation was observed in the presence of oxaprozin in CD40L-simulated monocytes. No significant activation/inhibition on protein phosphorylation in comparison with control medium was observed in the presence of oxaprozin alone (data not shown). These data suggest that oxaprozin treatment is associated with the inhibition of Akt and NF- $\kappa \mathrm{B}$ phosphorylation in CD40L-treated monocytes. Although crucial in CD40L-induced monocyte survival, ERK $1 / 2$ and p38 MAPK are not involved in oxaprozin-mediated proapoptotic effects.

3.4. Oxaprozin Proapoptotic Activity Is Associated with Caspase 3 Activation. We also investigated oxaprozin activity on CD40L-mediated caspase 3 regulation, as a well-known apoptotic pathway $[20,21]$. According to apoptosis experiments, oxaprozin treatment was associated with a significant increase of caspase 3 activity in CD40L-treated monocytes (Figure 4(a)). To further confirm the involvement of caspase 3 in oxaprozin-mediated proapoptotic activity, treatment with caspase 3 inhibitor blocked oxaprozin-induced apoptosis in CD40L-treated monocytes (Figure 4(b)). 


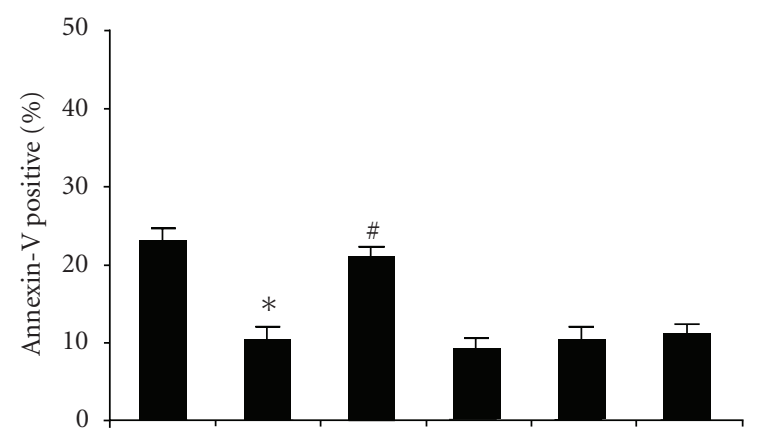

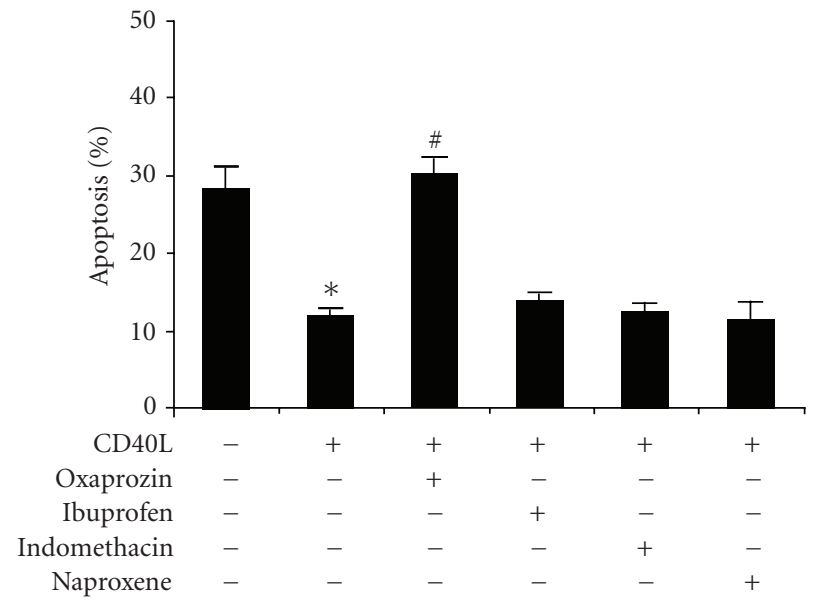

(a)

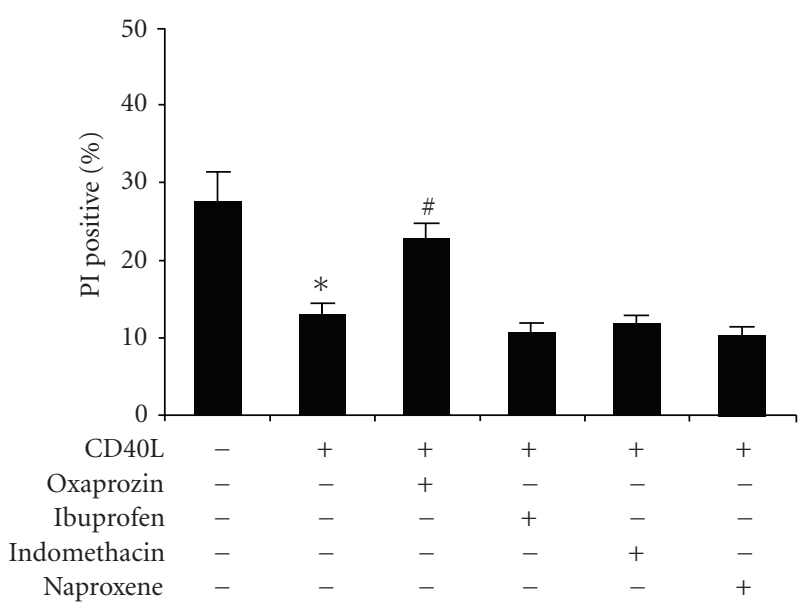

(b)

FIGURE 2: Oxaprozin-induced apoptosis is independent on COX inhibition. Monocytes were incubated for 48 hours with control medium or $200 \mathrm{ng} / \mathrm{mL}$ CD $40 \mathrm{~L}$ plus $1 \mu \mathrm{g} / \mathrm{mL}$ CD $40 \mathrm{~L}$ enhancer in the presence or absence of $100 \mu \mathrm{M}$ oxaprozin, $100 \mu \mathrm{M}$ ibuprofen, $100 \mu \mathrm{M}$ indomethacin, or $100 \mu \mathrm{M}$ naproxene. (a) Assessment of apoptosis by acridine-orange-stained slides under fluorescence microscopy. Data are express, as percentages of apoptotic cells on total number of cells counted (mean $\pm \mathrm{SEM}, n=4$; apoptosis in absence versus in presence of CD40L: ${ }^{*} P<.05$; apoptosis in presence of CD40L versus CD40L plus oxaprozin: ${ }^{\#} P<.05$; apoptosis in presence of CD40L versus CD40L plus other NSAIDs: N.S.). (b) Assessment of apoptosis by using Annexin V and Propidium Iodide (PI) binding assay (mean \pm SEM, $n=3$; apoptosis in absence versus in presence of CD40L: ${ }^{*} P<.05$; apoptosis in presence of CD40L versus CD40L plus oxaprozin: ${ }^{*} P<.05$; apoptosis in presence of CD40L versus CD40L plus other NSAIDs: N.S.).

\section{Discussion}

The induction of monocyte apoptosis rather than necrosis might represent a crucial approach to chronic inflammatory diseases, such as rheumatoid arthritis and atherosclerosis. In fact, in contrast to cells undergoing necrosis, the contents of those undergoing apoptosis are not released but cleared as apoptotic bodies by phagocytosis. On the other hand, necrotic cell death promotes inflammation, while apoptotic cell death results in an anti-inflammatory condition. Therefore, immune cell apoptosis is actually considered as a very promising anti-inflammatory therapeutic strategy [22]. On the other hand, CD40L represents one of the most important mediators in inflammatory processes modulating both infections and chronic diseases, including atherosclerosis, rheumatoid arthritis and transplantation [23-28]. CD40L has been already shown to prolong monocyte survival [9]. CD40 is also present on B cell, dendritic cell, monocyte, macrophage, mast cell, fibroblast, and endothelial cell membrane and regulates several immune functions, such as B cell response, antigen presenting cell activity, monocyte migration, and survival [29-31]. These activities resulted from the binding with two forms of CD40L. The first form is expressed on activated $\mathrm{T}$ and other immune cell membrane (CD154), while the second one is a soluble form, also called soluble CD40 ligand (sCD40L) [31]. Thus, given the pivotal role of monocytes and sCD40L in inflammatory diseases, we investigated the possible effect of the NSAIDs on CD40L-induced monocyte survival [32]. Among NSAIDs (drugs known for their potent COX inhibitory activity), only oxaprozin dose dependently increases apoptosis in CD40L-treated monocytes. This effect was observed at physiological concentrations achievable in inflamed tissues [33]. These data suggest that a possible molecular mechanism independent of COX inhibition might be associated with oxaprozin proapoptotic effect. To inhibit COX, we used NSAIDs at higher concentrations $(100 \mu \mathrm{M})$ than their previously reported $\mathrm{IC}_{50}$ for $\mathrm{COX}-1$ and -2 in intact cells [19]. In particular, $\mathrm{IC}_{50}$ of oxaprozin for human platelet COX-1 and for human inteleukin-1 $\beta$ stimulated 


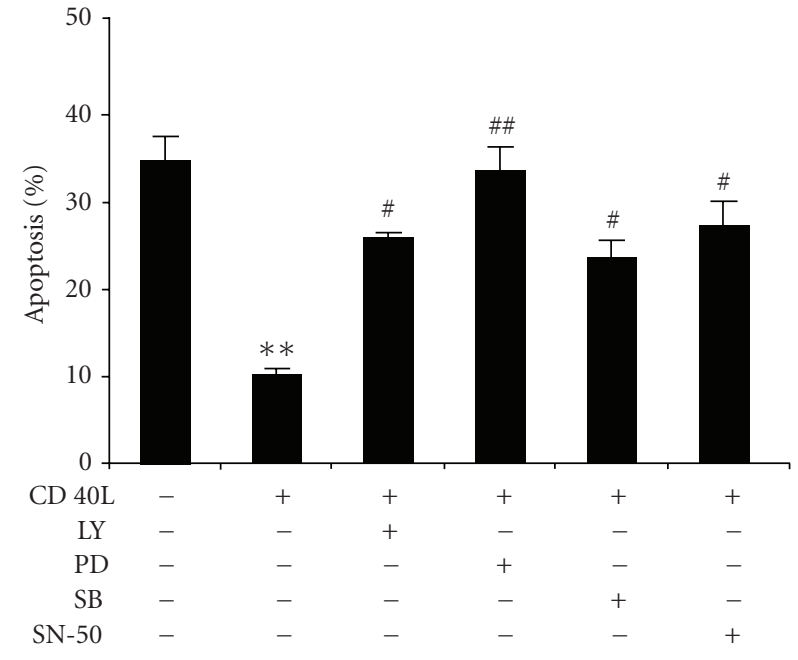

(a)
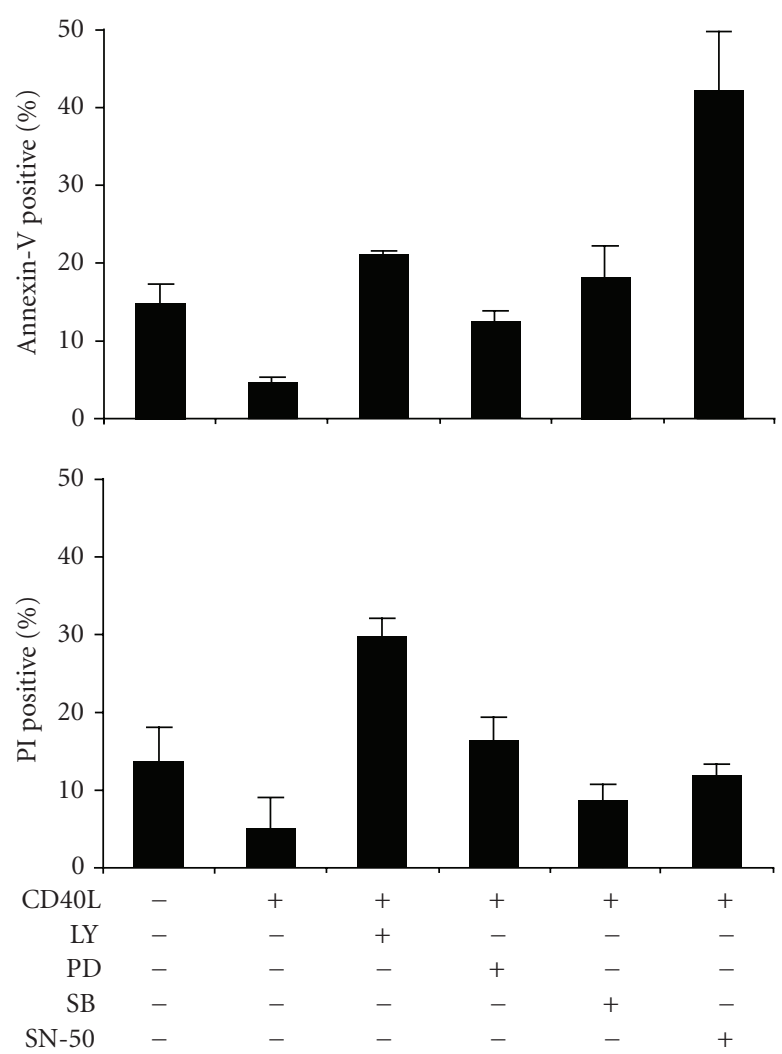

(b)

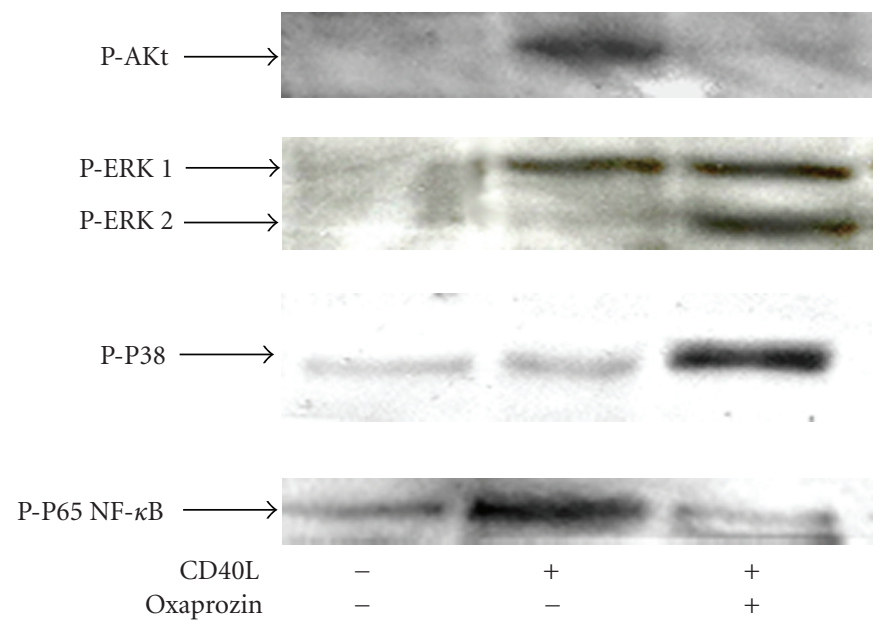

(c)

FIGURE 3: Oxaprozin-induced apoptosis involves the inhibition of CD40L-induced Akt and NF- $\kappa B$ phosphorylation. Monocytes were incubated for 48 hours with control medium or $200 \mathrm{ng} / \mathrm{mL}$ CD40L plus $1 \mu \mathrm{g} / \mathrm{mL}$ CD $40 \mathrm{~L}$ enhancer in the presence or absence of $50 \mu \mathrm{M}$ LY294002 (LY), $50 \mu \mathrm{M}$ PD98059 (PD), $1 \mu \mathrm{M}$ SB203580 (SB), or $20 \mu \mathrm{M}$ SN-50. (a) Assessment of apoptosis on cytopreps stained with acridine orange. Data are expressed as percentages of apoptotic cells on total number of cells counted (mean \pm SEM, $n=3$, apoptosis in absence versus in presence of CD40L: ${ }^{* *} P<.01$; apoptosis in presence of CD40L versus CD40L plus $\mathrm{PD}:{ }^{\#} P<.01$; apoptosis in presence of CD40L versus CD40L plus LY or SB or SN-50: ${ }^{P}<.05$ ). (b) Assessment of apoptosis using Annexin V and Propidium Iodide (PI) binding assay. Data are expressed as percentages of positive cells on total number of cells counted (mean $\pm \mathrm{SEM}, n=2$ ). (c) Monocytes were prencubated in the presence or absence of $100 \mu \mathrm{M}$ oxaprozin for 1 hour and then stimulated with control medium or $200 \mathrm{ng} / \mathrm{mL}$ CD40L plus $1 \mu \mathrm{g} / \mathrm{mL}$ CD40L enhancer for 15 minutes. Then, cells were lysed, and western blot analysis was performed. Results represent one of three experiments that yielded similar results. 


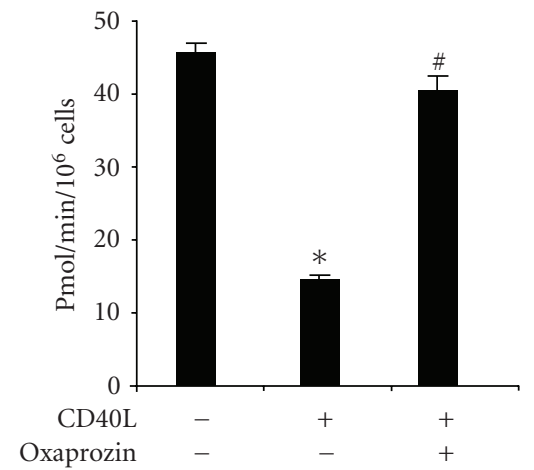

(a)

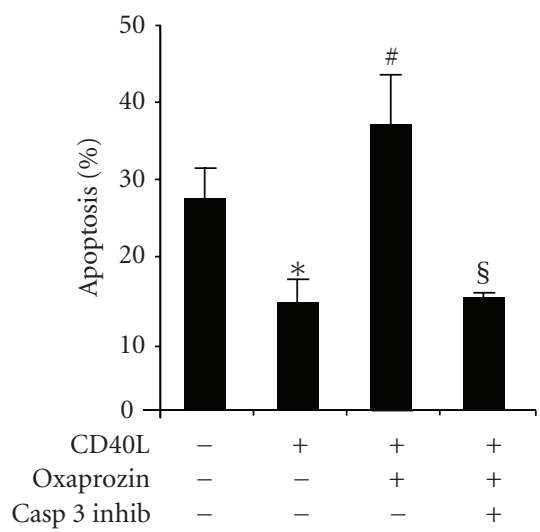

(b)

FIGURE 4: Oxaprozin-induced apoptosis of CD40L-treated monocytes involves the activation of caspase 3. (a) Monocytes were cultured for 48 hours with control medium or $200 \mathrm{ng} / \mathrm{mL} \mathrm{CD} 40 \mathrm{~L}$ plus $1 \mu \mathrm{g} / \mathrm{mL}$ CD $40 \mathrm{~L}$ enhancer in the presence or absence of $100 \mu \mathrm{M}$ of oxaprozin. Then cells were lysed, and caspase 3 activity was determined by spectrophotometer. Data are expressed as mean \pm SEM, $n=3$. (caspase 3 activity in absence versus in presence of CD40L: ${ }^{*} P<.05$; caspase 3 activity in presence of CD40L versus CD40L plus oxaprozin: ${ }^{\#} P<.05$ ). (b) Monocytes were incubated for 48 hours with control medium or $200 \mathrm{ng} / \mathrm{mL}$ CD $40 \mathrm{~L}$ plus $1 \mu \mathrm{g} / \mathrm{mL}$ CD $40 \mathrm{~L}$ enhancer in the presence or absence $100 \mu \mathrm{M}$ of oxaprozin and $50 \mu \mathrm{M}$ Ac-DEVD-CHO (caspase 3 inhibitor, CASP 3 INHIB). Data are expressed as mean \pm SEM, $n=3$ (apoptosis in absence versus in presence of CD40L: ${ }^{*} P<.05$; apoptosis in presence of CD40L versus CD40L plus oxaprozin: ${ }^{\#} P<.05$; apoptosis in presence of CD40L plus oxaprozin versus CD40L plus oxaprozin and caspase 3 inhibitor: ${ }^{\S} P<.05$ ).

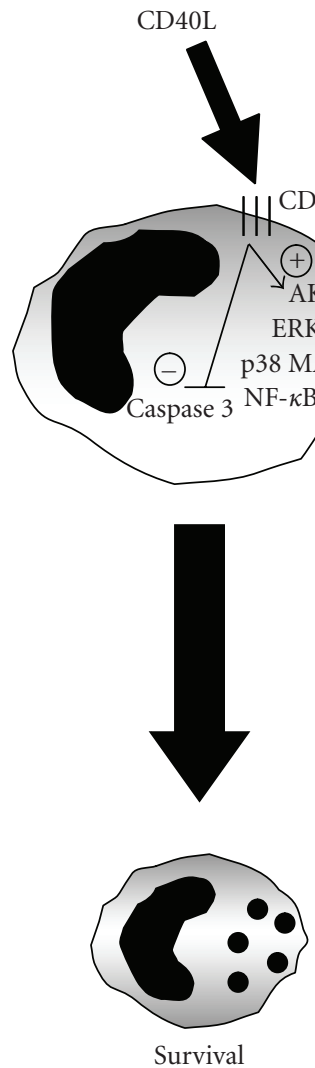

(a)
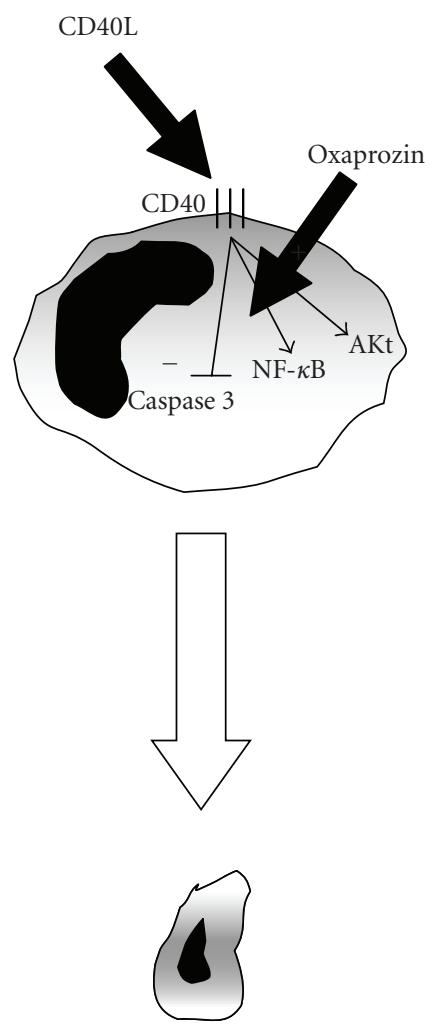

Apoptosis

(b)

FIGURE 5: (a) CD40L-induced monocyte survival is associated with Akt, ERK 1/2, P38 MAPK and NF- $\kappa$ B activation, and caspase 3 inhibition. (b) Oxaprozin-induced apoptosis in CD40L-treated monocytes is associated with the inhibition of Akt and NF- $\kappa \mathrm{B}$ and the activation of caspase 3 pathways. 
synovial cell COX-2 were, respectively, $2.2 \pm 0.56 \mu \mathrm{M}$ and $36 \pm 28 \mu \mathrm{M}$. These concentrations were similar to $\mathrm{IC}_{50}$ previously reported for the other NSAIDs in intact cells [19]. Therefore, although oxaprozin biological activities are commonly explained by the inhibition of COX, the lack of proapoptotic activity of other NSAIDs suggests that oxaprozin-induced increase of apoptosis in CD40L-treated monocytes was not mediated by the inhibition of COX. Then, we focused on COX-independent pathways [11]. Oxaprozin-mediated beneficial effect on monocyte apoptosis is associated with the inhibition of Akt and NF- $\kappa \mathrm{B}$ and the activation of caspase 3 pathways. This was suggested by two different findings: (1) CD40L-induced monocyte survival was associated with the activation of PI3K-Akt, p38 MAPK, $\mathrm{NF}-\kappa \mathrm{B}$, and ERK 1/2 [34-38], and the activation of all intracellular proteins was required to observe the increased survival; (2) oxaprozin-increased apoptosis of CD40Lstimulated monocytes was associated with the inhibition of Akt and NF- $\kappa$ B. Apoptosis experiments with intracellular protein inhibitors clearly indicate that each intracellular molecule represents a potential target for the pharmacological inhibition of CD40L-induced proinflammatory effects. Although the inhibition of NF- $\kappa \mathrm{B}$ was previously reported, the association between oxaprozin treatment and Akt inhibition in CD40L-stimulated monocytes represents a novel finding. Surprisingly, we also showed that oxaprozin treatment was associated with an increase of CD40L-induced ERK $1 / 2$ and p38 MAPK phosphorylation. No significant increase/inhibition in comparison with control medium was observed on ERK $1 / 2$ or p38 MAPK phosphorylation in the presence of oxaprozin alone. Thus, the pharmacological increase of the activation of these kinases represents an unexpected and novel finding, not completely understood. Synthetic anti-inflammatory drugs have been recently shown to increase MAPK activation in other cell types $[39,40]$. However, much remains to be clarified in this controversial "activatory" approach [41]. Thus, it could represent an interesting field for future investigations. Finally, the present article showed that oxaprozin treatment was associated with an increase of caspase 3 activation in CD40L-treated human monocytes. This has to be considered as a less specific mechanism of apoptosis induction. In fact, caspase 3 represents the common final pathway of apoptosis, in which converges several pathways [42] and might correspond to upstream oxaprozin-mediated events.

\section{Conclusions}

Oxaprozin has been shown as an effective drug in the clinical management of adult rheumatoid arthritis, osteoarthritis, ankylosing spondylitis, soft tissue disorders, and postoperative dental pain [43]. In particular, oxaprozin treatment showed better improvement in shoulder function and in the mental health item of the SF-36 quality of life component in comparison with diclofenac [44]. Beneficial effects of oxaprozin might be due to the high rate of accumulation in inflamed synovium in comparison with plasma and synovial fluid levels [33]. On the other hand, oxaprozin might be also a more active drug than other NSAIDs. Both antiinflammatory and analgesic properties of oxaprozin are mainly due to the potent inhibition of COX. However, oxaprozin-induced benefits might be also regulated by other COX-independent pathways [11]. This approach could partially explain the superior role of oxaprozin in comparison with other NSAIDs. The present paper shows that oxaprozin induced direct proapoptotic effects in CD40L-treated human primary monocytes independently of COX inhibition. The modulation of apoptotic mediators (such as Akt, NF- $\kappa \mathrm{B}$, and caspase 3) was associated with oxaprozin-mediated proapoptotic effects (Figure 5). Although highly speculative, these data support oxaprozin as a promising pharmacologic agent to suppress monocyte survival and their deleterious activities in chronic inflammatory disorders, such as rheumatoid arthritis.

\section{Acknowledgments}

This research was funded by EU FP7, Grant no. 201668, AtheroRemo and supported by a Grant from the Swiss National Science Foundation to Dr. F. Mach (no. 310030118245). There is no conflict of interest to be declared. Dr. Franco Dallegri received research funding from Helsinn Healthcare SA (Pambio-Noranco, Switzerland) as a part of contribution to the present work.

\section{References}

[1] G. K. Hansson and P. Libby, "The immune response in atherosclerosis: a double-edged sword," Nature Reviews Immunology, vol. 6, no. 7, pp. 508-519, 2006.

[2] C. Weber, A. Zernecke, and P. Libby, "The multifaceted contributions of leukocyte subsets to atherosclerosis: lessons from mouse models," Nature Reviews Immunology, vol. 8, no. 10, pp. 802-815, 2008.

[3] Z. Szekanecz and A. E. Koch, "Macrophages and their products in rheumatoid arthritis," Current Opinion in Rheumatology, vol. 19, no. 3, pp. 289-295, 2007.

[4] P. F. Bradfield, C. Scheiermann, S. Nourshargh, et al., "JAM-C regulates unidirectional monocyte transendothelial migration in inflammation," Blood, vol. 110, no. 7, pp. 2545-2555, 2007.

[5] A. Mantovani, A. Sica, and M. Locati, "New vistas on macrophage differentiation and activation," European Journal of Immunology, vol. 37, no. 1, pp. 14-16, 2007.

[6] S. Kumar and R. Jack, "Invited review: origin of monocytes and their differentiation to macrophages and dendritic cells," Journal of Endotoxin Research, vol. 12, no. 5, pp. 278-284, 2006.

[7] N. Takahashi, N. Udagawa, and T. Suda, "A new member of tumor necrosis factor ligand family, ODF/OPGL/ TRANCE/RANKL, regulates osteoclast differentiation and function," Biochemical and Biophysical Research Communications, vol. 256, no. 3, pp. 449-455, 1999.

[8] A. Dupoux, J. Cartier, S. Cathelin, R. Filomenko, E. Solary, and D.-D. Laurence, "cIAP1-dependent TRAF2 degradation regulates the differentiation of monocytes into macrophages and their response to CD40 ligand," Blood, vol. 113, no. 1, pp. 175-185, 2009.

[9] P. A. Kiener, P. Moran-Davis, B. M. Rankin, A. F. Wahl, A. Aruffo, and D. Hollenbaugh, "Stimulation of CD40 with purified soluble gp39 induces proinflammatory responses in 
human monocytes," The Journal of Immunology, vol. 155, no. 10, pp. 4917-4925, 1995.

[10] N. C. Kaneider, A. Kaser, H. Tilg, G. Ricevuti, and C. J. Wiedermann, "CD40 ligand-dependent maturation of human monocyte-derived dendritic cells by activated platelets," International Journal of Immunopathology and Pharmacology, vol. 16, no. 3, pp. 225-231, 2003.

[11] L. Ottonello, M. Bertolotto, F. Montecucco, G. Bianchi, and F. Dallegri, "Delayed apoptosis of human monocytes exposed to immune complexes is reversed by oxaprozin: role of the Akt/I $\kappa \mathrm{B}$ kinase/nuclear factor $\kappa \mathrm{B}$ pathway," British Journal of Pharmacology, vol. 157, no. 2, pp. 294-306, 2009.

[12] L. Ottonello, M. Bertolotto, F. Montecucco, P. Dapino, and F. Dallegri, "Dexamethasone-induced apoptosis of human monocytes exposed to immune complexes. Intervention of CD95-and XIAP-dependent pathways," International Journal of Immunopathology and Pharmacology, vol. 18, no. 3, pp. 403$415,2005$.

[13] I. Nicoletti, G. Migliorati, M. C. Pagliacci, and C. Riccardi, "A rapid and simple method for measuring thymocyte apoptosis by propidium iodide staining and flow cytometry," Journal of Immunological Methods, vol. 139, no. 2, pp. 271-279, 1991.

[14] L. Ottonello, F. Montecucco, M. Bertolotto, et al., "CCL3 $(\mathrm{MIP}-1 \alpha)$ induces in vitro migration of GM-CSF-primed human neutrophils via CCR5-dependent activation of ERK 1/2," Cellular Signalling, vol. 17, no. 3, pp. 355-363, 2005.

[15] L. Ottonello, G. Frumento, N. Arduino, P. Dapino, G. Tortolina, and F. Dallegri, "Immune complex stimulation of neutrophil apoptosis: investigating the involvement of oxidative and nonoxidative pathways," Free Radical Biology and Medicine, vol. 30, no. 2, pp. 161-169, 2001.

[16] H. Nielsen and J. Bennedsen, "Effect of nonsteroidal antiinflammatory drugs on human monocyte chemotaxis in vitro," Immunopharmacology, vol. 5, no. 4, pp. 259-265, 1983.

[17] C. Rae, S. Langa, S. J. Tucker, and D. J. MacEwan, "Elevated NF- $\kappa$ B responses and FLIP levels in leukemic but not normal lymphocytes: reduction by salicylate allows TNF-induced apoptosis," Proceedings of the National Academy of Sciences of the United States of America, vol. 104, no. 31, pp. 12790-12795, 2007.

[18] S. Kawai, S. Nishida, M. Kato, et al., "Comparison of cyclooxygenase-1 and -2 inhibitory activities of various nonsteroidal anti-inflammatory drugs using human platelets and synovial cells," European Journal of Pharmacology, vol. 347, no. 1, pp. 87-94, 1998.

[19] J. A. Mitchell, P. Akarasereenont, C. Thiemermann, R. J. Flower, and J. R. Vane, "Selectivity of nonsteroidal antiinflammatory drugs as inhibitors of constitutive and inducible cyclooxygenase," Proceedings of the National Academy of Sciences of the United States of America, vol. 90, no. 24, pp. 11693-11697, 1993.

[20] E. S. Wintergerst, J. Jelk, C. Rahner, and R. Asmis, "Apoptosis induced by oxidized low density lipoprotein in human monocyte-derived macrophages involves CD36 and activation of caspase-3," European Journal of Biochemistry, vol. 267, no. 19, pp. 6050-6058, 2000.

[21] R. J. Fahy, A. I. Doseff, and M. D. Wewers, "Spontaneous human monocyte apoptosis utilizes a caspase-3-dependent pathway that is blocked by endotoxin and is independent of caspase-1," The Journal of Immunology, vol. 163, no. 4, pp. 1755-1762, 1999.

[22] H. Liu and R. M. Pope, "Apoptosis in rheumatoid arthritis: friend or foe," Rheumatic Disease Clinics of North America, vol. 30, no. 3, pp. 603-625, 2004.
[23] I. S. Grewal, P. Borrow, E. G. Pamer, M. B. Oldstone, and R. A. Flavell, "The CD40-CD154 system in anti-infective host defense," Current Opinion in Immunology, vol. 9, no. 4, pp. 491-497, 1997.

[24] J. D. Laman, M. Van Meurs, B. J. G. L. de Smet, and A. Schoneveld, "CD40-CD40L interactions in atherosclerosis," Immunology Today, vol. 18, no. 6, pp. 272-277, 1997.

[25] U. Schonbeck and P. Libby, "CD40 signaling and plaque instability," Circulation Research, vol. 89, no. 12, pp. 10921103, 2001.

[26] F. H. Durie, R. A. Fava, T. M. Foy, A. Aruffo, J. A. Ledbetter, and R. J. Noelle, "Prevention of collagen-induced arthritis with an antibody to gp39, the ligand for CD40," Science, vol. 261, no. 5126, pp. 1328-1330, 1993.

[27] D. C. Parker, D. L. Greiner, N. E. Phillips, et al., "Survival of mouse pancreatic islet allografts in recipients treated with allogeneic small lymphocytes and antibody to CD40 ligand," Proceedings of the National Academy of Sciences of the United States of America, vol. 92, no. 21, pp. 9560-9564, 1995.

[28] F. Montecucco and F. Mach, "Common inflammatory mediators orchestrate pathophysiological processes in rheumatoid arthritis and atherosclerosis," Rheumatology, vol. 48, no. 1, pp. 11-22, 2009.

[29] R. D. Stout and J. Suttles, "The many roles of CD40 in cellmediated inflammatory responses," Immunology Today, vol. 17, no. 10, pp. 487-492, 1996.

[30] C. Van Kooten and J. Banchereau, "CD40-CD40 ligand: a multifunctional receptor-ligand pair," Advances in Immunology, vol. 61, pp. 1-77, 1996.

[31] I. S. Grewal and R. A. Flavell, "CD40 and CD154 in cellmediated immunity," Annual Review of Immunology, vol. 16, pp. 111-135, 1998.

[32] F. Dallegri, M. Bertolotto, and L. Ottonello, "A review of the emerging profile of the anti-inflammatory drug oxaprozin," Expert Opinion on Pharmacotherapy, vol. 6, no. 5, pp. 777-785, 2005.

[33] P. A. Todd and R. N. Brogden, "Oxaprozin: a preliminary review of its pharmacodynamic and pharmacokinetic properties, and therapeutic efficacy," Drugs, vol. 32, no. 4, pp. 291$312,1986$.

[34] S. Chakrabarti, P. Blair, and J. E. Freedman, "CD40-40L signaling in vascular inflammation," Journal of Biological Chemistry, vol. 282, no. 25, pp. 18307-18317, 2007.

[35] J.-C. Yan, Z.-G. Wu, X.-T. Kong, R.-Q. Zong, and L.-Z. Zhang, "Effect of CD40-CD40 ligand interaction on diacylglycerolprotein kinase $\mathrm{C}$ signal transduction pathway and intracellular calcium in cultured human monocytes," Acta Pharmacologica Sinica, vol. 24, no. 7, pp. 687-691, 2003.

[36] A. D. Foey, M. Feldmann, and F. M. Brennan, "CD40 ligation induces macrophage IL-10 and TNF- $\alpha$ production: differential use of the PI3K and p42/44 MAPK-pathways," Cytokine, vol. 16, no. 4, pp. 131-142, 2001.

[37] P. Revy, C. Hivroz, G. Andreu, et al., "Activation of the Janus kinase 3-STAT5a pathway after CD40 triggering of human monocytes but not of resting B cells," The Journal of Immunology, vol. 163, no. 2, pp. 787-793, 1999.

[38] C. Léveillé, M. Bouillon, W. Guo, et al., "CD40 ligand binds to $\alpha 5 \beta 1$ integrin and triggers cell signaling," Journal of Biological Chemistry, vol. 282, no. 8, pp. 5143-5151, 2007.

[39] R. Merla, Y. Ye, Y. Lin, et al., "The central role of adenosine in statin-induced ERK1/2, Akt, and eNOS phosphorylation," American Journal of Physiology, vol. 293, no. 3, pp. H1918H1928, 2007. 
[40] F. Montecucco, S. Lenglet, V. Braunersreuther, et al., " $\mathrm{CB}_{2}$ cannabinoid receptor activation is cardioprotective in a mouse model of ischemia/reperfusion," Journal of Molecular and Cellular Cardiology, vol. 46, no. 5, pp. 612-620, 2009.

[41] C. Tsatsanis, A. Androulidaki, M. Venihaki, and A. N. Margioris, "Signalling networks regulating cyclooxygenase-2," International Journal of Biochemistry and Cell Biology, vol. 38, no. 10, pp. 1654-1661, 2006.

[42] P. Hofman, "Molecular regulation of neutrophil apoptosis and potential targets for therapeutic strategy against the inflammatory process," Current Drug Targets: Inflammation and Allergy, vol. 3, no. 1, pp. 1-9, 2004.

[43] W. F. Kean, "Oxaprozin: kinetic and dynamic profile in the treatment of pain," Current Medical Research and Opinion, vol. 20, no. 8, pp. 1275-1277, 2004.

[44] B. Heller and R. Tarricone, "Oxaprozin versus diclofenac in NSAID-refractory periarthritis pain of the shoulder," Current Medical Research and Opinion, vol. 20, no. 8, pp. 1279-1290, 2004. 

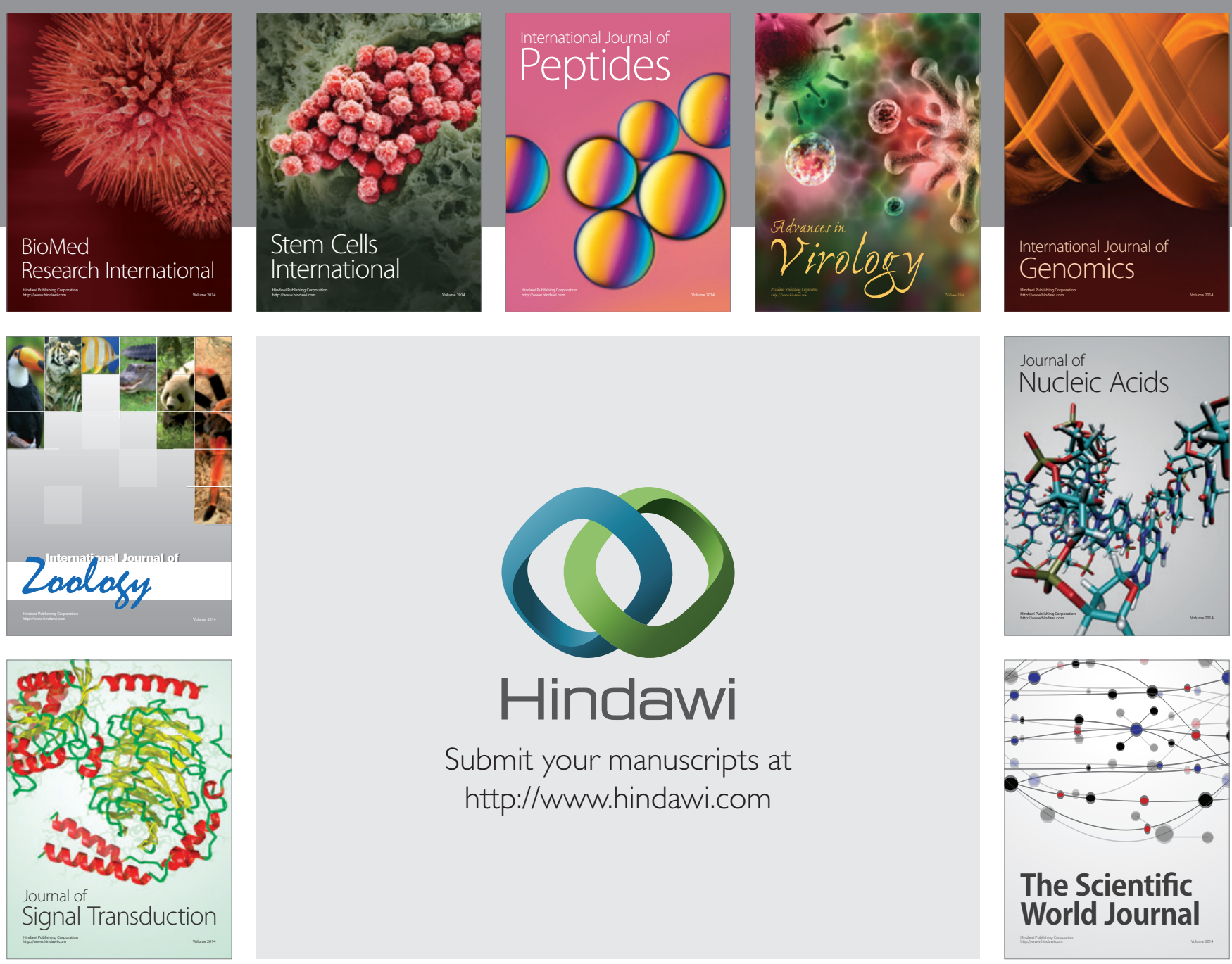

Submit your manuscripts at

http://www.hindawi.com
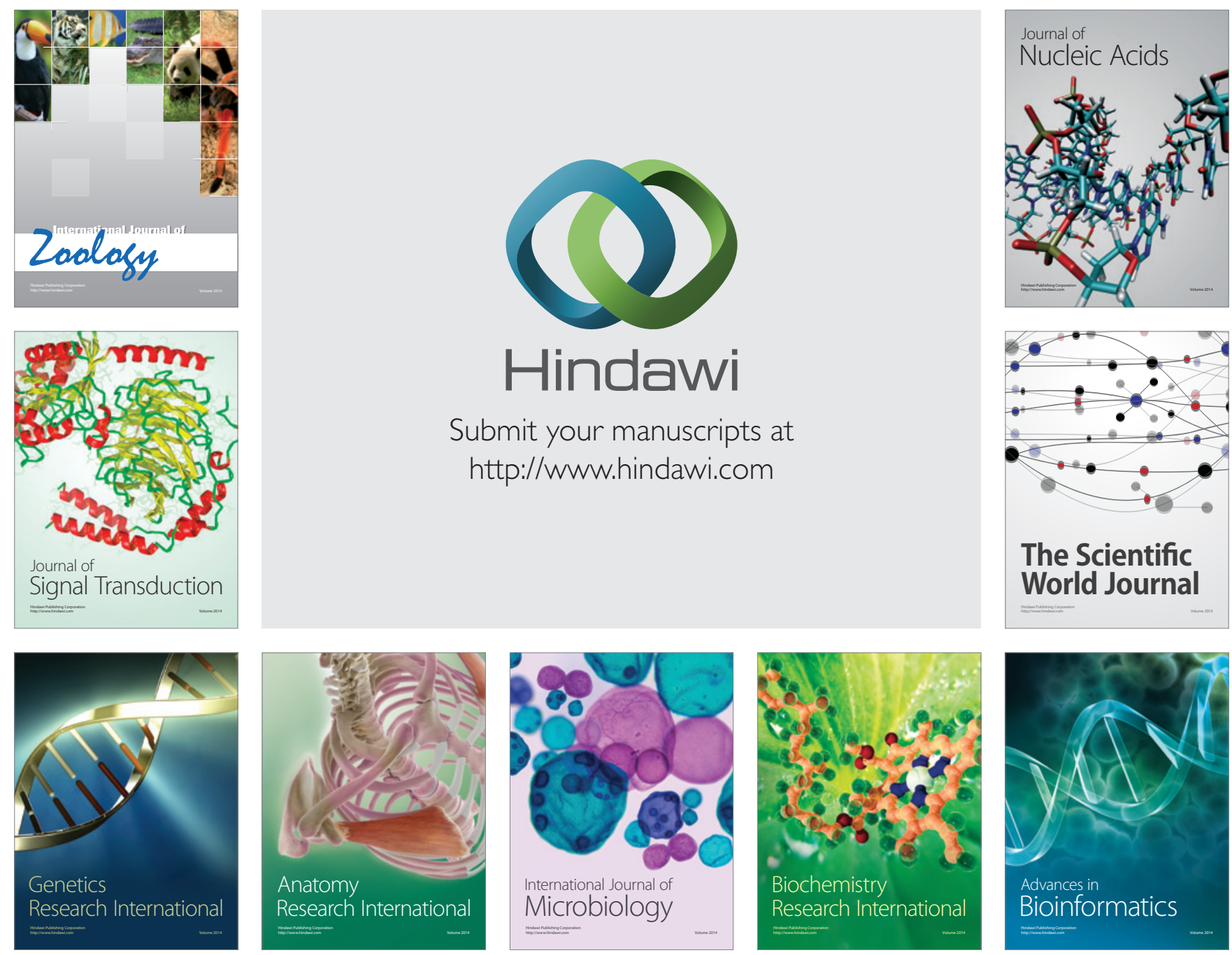

The Scientific World Journal
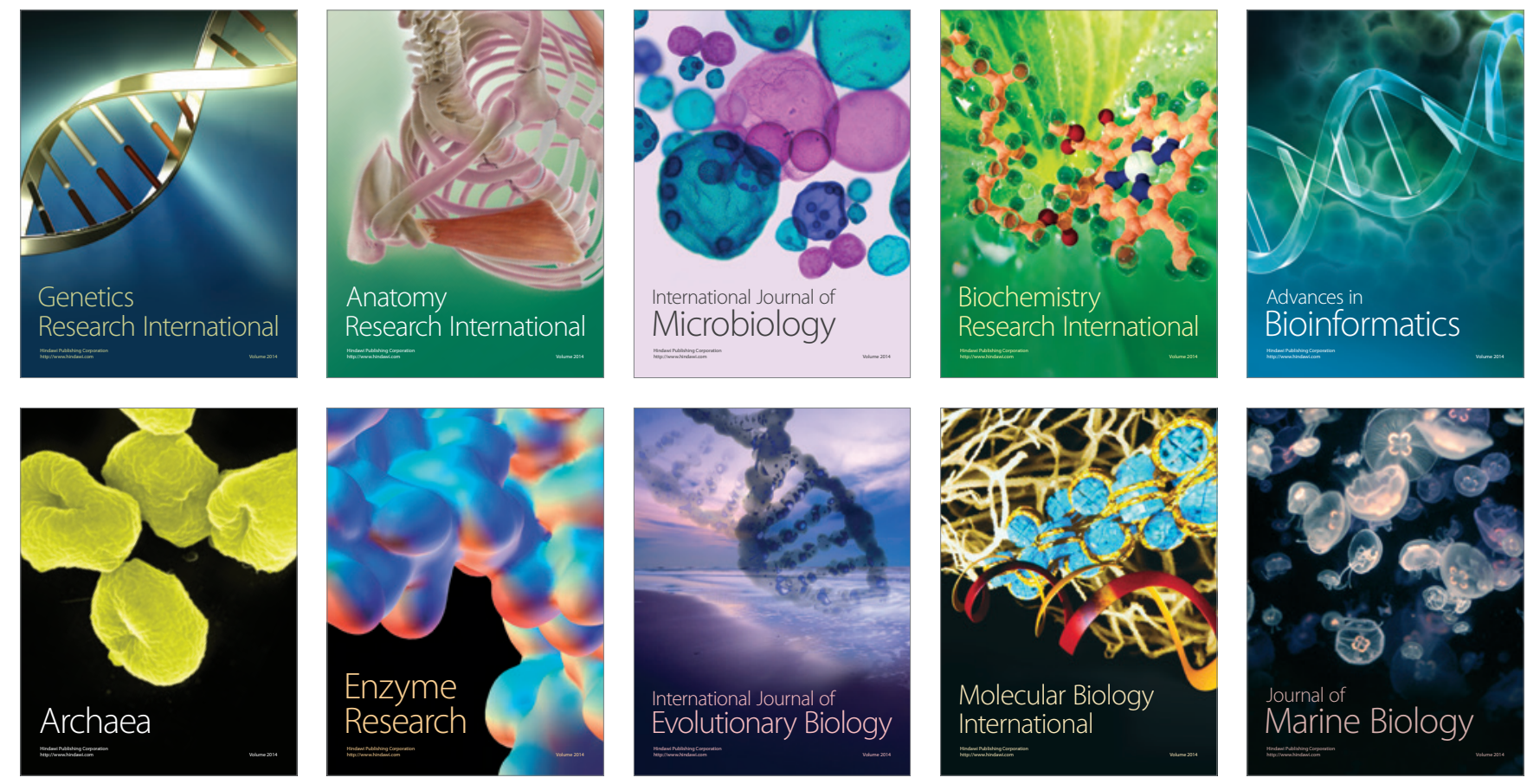